Brazilian Journal

of Chemical

ISSN 0104-6632

Engineering

\title{
EXPERIMENTAL STUDY ON THE COLLABORATIVE DRAG REDUCTION PERFORMANCE OF A SURFACTANT SOLUTION IN GROOVED CHANNELS
}

\author{
Chonghai Huang and Jinjia Wei* \\ State Key Laboratory of Multiphase Flow in Power Engineering, \\ Xi' an Jiaotong University, No. 28 Xianning West Road, Xi'an 710049, China. \\ Phone: + 86-29-82664462 \\ E-mail: jjwei@mail.xjtu.edu.cn
}

(Submitted: April 15, 2015 ; Revised: October 9, 2015 ; Accepted: December 22, 2015)

\begin{abstract}
Turbulence with a relatively larger vortex is obtained in drag-reducing surfactant solution, which provides an excellent condition for the application of small scale grooves. In this work, the coupling drag reduction performance of surfactant solution and grooves was experimentally investigated to explore the complementary possibility between their drag reduction mechanisms. The cationic surfactant cetyltrimethyl ammonium chloride (CTAC) mixed with the counterion salt sodium salicylate (NaSal) was experimented in smooth or grooved channel, respectively, at the mass concentrations of 50-150 ppm. It was found that the surfactant solutions gave more effective drag reduction in the grooved channel by the interaction between the "restriction effect" and "peak effect" of grooves. Moreover, the critical temperature and critical Reynolds number of the surfactant solution were smaller in the grooved channel, and the friction factor in the grooved channel increased much more rapidly than that in the smooth channel when Re is larger than a critical value.

Keywords: Turbulence; Drag reduction; Surfactant; Groove; Interaction.
\end{abstract}

\section{INTRODUCTION}

In pipe or channel flows, a great part of the energy loss is caused by skin friction. The focus of the current research is therefore to find the solution of reducing skin friction drag. Drag reduction can be achieved either by adding small amounts of additives such as polymers (Al-Wahaibi et al., 2013), surfactants (Li et al., 2006), particles (Pang et al., 2011), etc., which can alter the fluid composition and then affect its flow resistance, or by using passive devices such as grooves (Quintavalla et al., 2013) or superhydrophobic coatings (Moaven et al., 2013) which directly affect the flow without altering the fluid.

For additives, since the discovery of Toms' effect (Toms, 1948; Mysels, 1949), reduction of turbulent friction can be achieved by adding drag-reducing additives to save the pumping power in fluid transportation. Polymers initially used as drag-reducing additives could reduce the friction drag by up to $80 \%$. One successful application of drag reduction by polymers was the Trans-Alaska crude oil transportation pipeline, the flow capacity of which increased by $15 \%$ with the same pumping power (Burger et al., 1982). However, polymer solutions are strongly affected by mechanical degradation, which consequently shortens their lifetime of drag reduction effectiveness and further limits their application in a long-term circular pipeline. Surfactants developed in the last three decades could also reduce the frictional drag by $60-80 \%$ and are less vulnerable to mechanical degradation than the former (Ohlen-

*To whom correspondence should be addressed 
dorf et al., 1986; Bewersdorff and Ohlendorf, 1988). Therefore, surfactants are now used as the efficient drag-reducing additives in the application.

Although the drag-reducing mechanism of surfactants is still imperfectly understood, some physical insights have emerged. Debye and Anacker (1951) found that the micelle structures can change from spherical micelles to rod-like or wormlike micelles with increasing concentration above the Critical Micelle Concentration (CMC). Rehage et al. (1986) reported that the apparent viscosity of dilute surfactant solutions increased significantly by increasing shear stress. This phenomenon was called a shearthickening transition, resulting from the formation of shear-induced structures (SIS). Kawaguchi et al. (2002) performed a study on the spatial structure of turbulence by PIV. They found that the formation of a turbulent vortex was inhibited and that the scale of the vortex became larger when the surfactant was added to water. This suggested that a turbulent environment with a relatively larger vortex was obtained and thus provided an excellent condition for application of other drag-reducing methods related to the scale of the turbulent vortex. Recently, Hadri et al. (2011) discovered that the interaction between the surfactant chains and the turbulent structure was related to the temperature and concentration, namely, only in a certain range of temperature and concentration could the surfactants suppress turbulent vortices.

Among passive drag-reducing devices, longitudinal microgrooves have been extensively studied by Walsh and his co-workers (Walsh, 1980; Walsh, 1982; Walsh and Lindemann, 1984). They found first that triangular grooves achieved the optimal drag reduction effect compared with the smooth surface when the height of the groove equaled its spacing. Walsh originally considered the grooves as "fences" which isolated the low speed streaks near the wall, and then inhibited the momentum transfer. Bechert et al. (1986) and Choi (1990) proposed that grooves could restrain the spanwise motions of streamwise vortex-pairs, resulting in weaker bursts and lower shear stresses. Koury and Virk (1995) also obtained the same results as Bechert et al. (1986). They examined the V-grooves and found that grooves could reduce turbulent kinetic energy exchange and overall surface friction by impeding the formation of low speed streaks and the spanwise motions of streamwise vortices, though they increased the surface area. Recently, Chamorro et al. (2013) suggested that, because the dragreducing effect of grooves was dependent on their size, it is better that the scale of grooves $s^{+}\left(=u_{\tau} s / v\right.$, where $u_{\tau}$ is the frictional velocity for water in the smooth channel) matches up with the turbulent vortex scale to ensure the drag reduction effectiveness.
With the analysis of the existing drag-reducing mechanism of surfactants and microgrooves, the drag-reducing performance of the microgroove being dependent on the scale of the turbulent vortex points to a restriction of the motions of turbulent vortices. Moreover, drag-reducing surfactant solutions can form a larger vortex environment, which means that their mechanisms might be complementary. However, there is little information available on the coupling effect between surfactants and grooves. Other researchers have carried out similar studies on the interaction between polymers and grooves (Koury and Virk, 1995). The coupling applications of surfactants and grooves would be better than that of polymers and grooves because of the irreversible mechanical degradation of polymers, but their results may only be used as a reference. For this reason, the purpose of this study is to examine the coupling drag reduction effect of surfactants and grooves and to verify the possibility of their complementary mechanisms.

\section{EXPERIMENTAL}

\section{Test Facility}

The experiments were performed on a closed loop shown schematically in Figure 1. The system consists of a storage tank, a stainless steel centrifugal pump, a settling chamber, a 2D channel, a diffuser and other necessary elements. The fluid temperatures are controlled by a $6 \mathrm{~kW}$ heater installed in the storage tank, and the accuracy of temperature is $\pm 0.1 \mathrm{~K}$. The low flow rate $\left(0.7-3 \mathrm{~m}^{3} / \mathrm{h}\right)$ and high flow rate $(>3$ $\mathrm{m}^{3} / \mathrm{h}$ ) are measured respectively by two parallel electromagnetic flowmeters (measuring accuracy of $0.001 \mathrm{~m}^{3} / \mathrm{h}$ and $0.01 \mathrm{~m}^{3} / \mathrm{h}$, respectively). The flow rates are adjusted by the motor frequency of a stainless steel centrifugal pump. The pressure drop of the test channel is measured by two pressure taps with a distance of $1.1 \mathrm{~m}$, using a differential pressure transmitter (measuring range of $0-10 \mathrm{kPa}$ and measuring accuracy of $10 \mathrm{~Pa}$ ).

The system was circulated by the pump. The aqueous solution was firstly supplied to the chamber, then passed through the filter to remove the foam, and flowed finally into the 2D channel through the honeycomb. The $45 \mathrm{~mm}$ long honeycomb with $8 \times 8 \mathrm{~mm}^{2}$ rectangular openings was used to remove the large eddies. The 2D channel of $10 \mathrm{~mm}$ height, $125 \mathrm{~mm}$ width and $3 \mathrm{~m}$ length consisted of a fully developed section and a test section. Each section was $1.5 \mathrm{~m}$. The aspect ratio of the 2D channel was more than 7 to insure a two-dimensional flow in the center of the spanwise distance. The fully developed section was 
long enough to ensure that the fluid in the test section developed a full turbulence. The test section could be replaced by the smooth channel or the grooved channel.

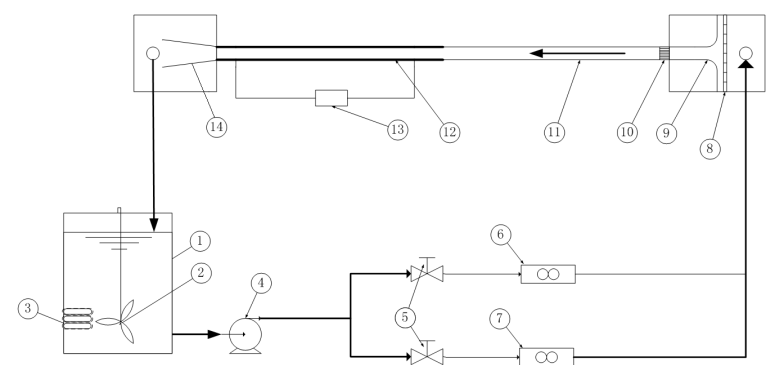

Figure 1: Schematic of the experimental system. (1) Storage tank; (2) stirrer; (3) heater; (4) stainless steel centrifugal pump; (5) valve; (6), (7) electromagnetic flowmeter; (8) filter; (9) contraction; (10) honeycomb; (11) 2D channel; (12) test section; (13) differential pressure transmitter; (14) diffuser.

\section{Microgrooves}

A 2D grooved channel consisting of two longitudinal microgroove surfaces (upper and lower surfaces) was examined in the experiment. The longitudinal microgroove surfaces are manufactured directly with a milling machine. The cross-section of the microgrooves made of transparent acrylic resin is shown in Figure 2. As shown in Figure 2, $s$ and $h$ represented the spacing and the depth of the grooves, respectively. $\alpha$ was the angle of the groove tip. Half of the depth of the groove was defined as the datum plane, and two grooved surfaces constituted the $2 \mathrm{D}$ grooved channel of $H=10 \mathrm{~mm}$. The grooves of $s=0.7 \mathrm{~mm} h=0.4 \mathrm{~mm}$, $\alpha=53$ were investigated in the experiment.

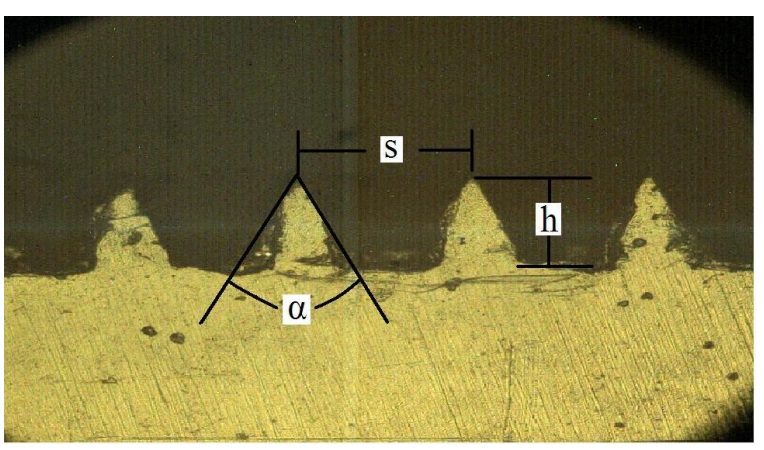

Figure 2: Schematic of the microgrooves.

\section{Surfactant}

The cationic surfactant used in this study was cetyl trimethyl ammonium chloride (CTAC) with the chemical formula of $\mathrm{C}_{16} \mathrm{H}_{33} \mathrm{~N}\left(\mathrm{CH}_{3}\right)_{3} \mathrm{Cl}$ and a molecu- lar weight of $320.0 \mathrm{~g} / \mathrm{mol}$. The counter-ion salt sodium salicylate (NaSal) with the chemical formula of $\mathrm{C}_{7} \mathrm{H}_{5} \mathrm{NaO}_{3}$ and a molecular weight of $160.1 \mathrm{~g} / \mathrm{mol}$ was used in this study with the same weight concentration as that of CTAC. The solvent was tap water and the surfactant solution is represented by CTAC concentration. Several mass concentrations $(50,70$, $100,150 \mathrm{ppm}$ ) of CTAC solution were tested at different temperatures and Reynolds numbers in the experiment.

\section{Data Processing}

The Fanning friction factor $C_{f}$ was defined as:

$C_{f}=\frac{2 \tau_{\mathrm{w}}}{\rho U_{\mathrm{b}}{ }^{2}}=\frac{\Delta P H W}{\rho U_{b}^{2}(H+W) L}$

where $\tau_{\mathrm{w}}$ is the wall shear stress, $\rho$ the density of solvent, $U_{b}$ the bulk mean velocity, $\Delta P$ the pressure drops over a certain streamwise distance $L$ of two pressure taps, $H$ and $W$ the height and width of $2 \mathrm{D}$ channel, respectively.

The drag reduction rates of grooves without surfactant, surfactant and the combination of them were defined respectively as:

$$
\begin{aligned}
& D R_{g} \%=\frac{C_{f}-C_{f g}}{C_{f}} \times 100 \% \\
& D R_{s} \%=\frac{C_{f}-C_{f s}}{C_{f}} \times 100 \% \\
& D R_{g s} \%=\frac{C_{f}-C_{f g s}}{C_{f}} \times 100 \%
\end{aligned}
$$

where $C_{f}$ is the Fanning friction factor of water in a smooth channel, and subscripts $\mathrm{g}, \mathrm{s}$ and gs represent the conditions of groove, surfactant and both of them, respectively.

Because the spanwise width of the $2 \mathrm{D}$ channel is much larger than the height, the Reynolds number was determined as follows:

$$
\operatorname{Re}=\frac{U_{b} * H}{v}
$$

where $v$ is the kinetic viscosity of solvent, depending on the bulk temperature of the solution.

\section{Uncertainty Analysis}

In the present study, the measurement uncertainty of $C_{f i}$ and DR can be estimated as follows: 


$$
\frac{\Delta_{C_{f i}}}{C_{f i}} \approx \sqrt{\left(\frac{\Delta_{\Delta P}}{\Delta P}\right)^{2}+\left(\frac{2 \cdot \Delta_{U_{b}}}{U_{b}}\right)^{2}+\left[\frac{W \cdot \Delta_{H}}{H(H+W)}\right]^{2}+\left[\frac{H \cdot \Delta_{W}}{W(H+W)}\right]^{2}+\left(\frac{\Delta_{\rho}}{\rho}\right)^{2}+\left(\frac{\Delta_{L}}{L}\right)^{2}}
$$

$\Delta_{D R} \approx \frac{\Delta_{C_{f i}}}{C_{f}}$

where $C_{f i}$ can be replaced with $C_{f g}, C_{f s}$ and $C_{f g s}$ mentioned above. $\Delta$ represents the uncertainty of the physical quantity.

\section{RESULTS AND DISCUSSION}

\section{Drag Reduction Performance}

The drag reduction curves of $70 \mathrm{ppm}$ CTAC solution in the smooth and grooved channels are respectively presented in Figure 3. The Fanning friction factor $C_{f}$ and Reynolds number Re are calculated by Eqs. (1) and (5) respectively. The Dean line (Dean, 1978), which was appropriate for the turbulent flow of a Newtonian fluid, and the Zakin line (Zakin et al., 1996), which represented the maximum drag reduction asymptote of surfactant, are cited respectively for comparison in the following figures. In order to guarantee the correctness of the experimental results, all experiments are repeated at least two times and the results are an average of them. As shown in Figure 3(a), the friction factor for water obeys Dean's (1978) experimental formula, indicating the experimental system is reliable. Moreover, it can also be seen from Figure 3 that the surfactant solutions in both the smooth and grooved channels show excellent drag reduction effectiveness compared with water. For a certain temperature, for example 20 ${ }^{\circ} \mathrm{C}$, the friction factor increases at $\mathrm{Re}=17000$ (seen from Figure 3(a)), which is defined as the critical Reynolds number $\mathrm{Re}_{\mathrm{c}}$. Similar phenomena occur at other temperatures. Figures. 3 and 4 show that the critical Reynolds numbers for both the smooth and grooved channels firstly increase with increasing temperature, then decrease as temperature continues to rise. The reason is that, for surfactant solutions, the shear-induced structure (SIS) could be formed under the action of shear stress, which was perceived by many researchers as the drag-reducing mechanism of surfactants (Zhou et al., 2011; Qi et al., 2011). Furthermore, the Brownian motions of molecule are enhanced as temperature increases. So is the capacity to form the shear-induced structure. Meanwhile, it indicates that the ability to resist shear failure is stronger and that the critical Reynolds number rises. However, when the temperature exceeds a certain degree (defined as the critical temperature $T_{c}$ ), the Brownian motion is more severe, and then the shearinduced structures are destroyed (Hadri et al., 2011), resulting in a decrease of the Reynolds number. Wei et al. (2012) also discovered the similar phenomena of the critical temperature and the critical Reynolds number in surfactant solutions. Similar phenomena can also be found in the results of $100 \mathrm{ppm}$ and 150 ppm CTAC solutions, thus we will not repeat here.

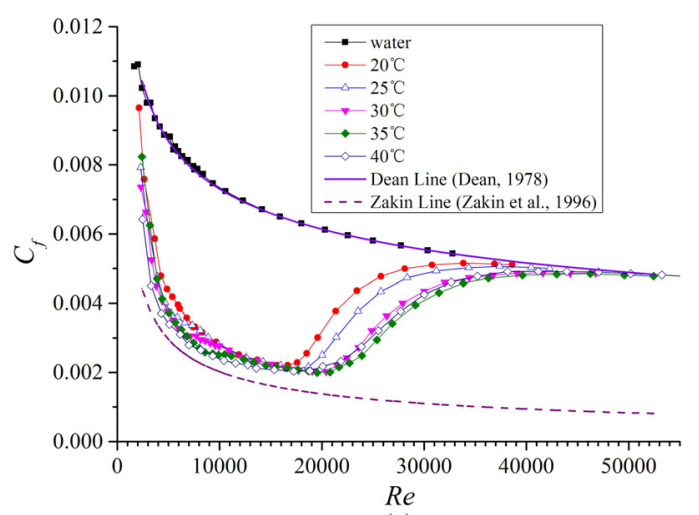

(a)

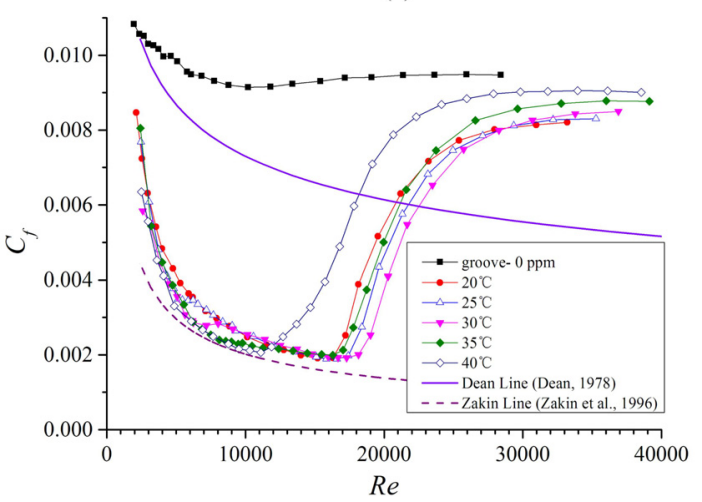

(b)

Figure 3: Drag-reducing performance of $70 \mathrm{ppm}$ CTAC. (a) Smooth channel. (b) Grooved channel. The Dean line and Zakin line are from Dean (1978) and Zakin et al. (1996), respectively.

In order to compare more directly the relationships between temperatures, and the critical Reynolds numbers for different concentrations of CTAC solutions in the smooth and grooved channels respectively, some relevant data are extracted from Figure 3 and the results for $100 \mathrm{ppm}$ and $150 \mathrm{ppm}$ CTAC solu- 
tions to plot Figure 4. It can be obviously seen that, at the same concentration, the critical Reynolds numbers and critical temperatures of surfactant solutions in the grooved channel are smaller than those in the smooth channel and that the difference of the critical Reynolds numbers between smooth and grooved channel increases with temperature. Because the shear stress at grooved tips is larger than that on the smooth surface at the same Reynolds number (Goldstein et al., 1995; Zhang et al., 2011), here we call this phenomenon the "peak effect" of grooves. The shear-induced structures (SIS) are destroyed more easily by the larger shear stress, resulting in smaller critical Reynolds numbers and critical temperatures in the grooved channel. Moreover, the increase in the difference of the critical Reynolds numbers is caused by the increasing influence of temperature on the thermal motions of molecule.

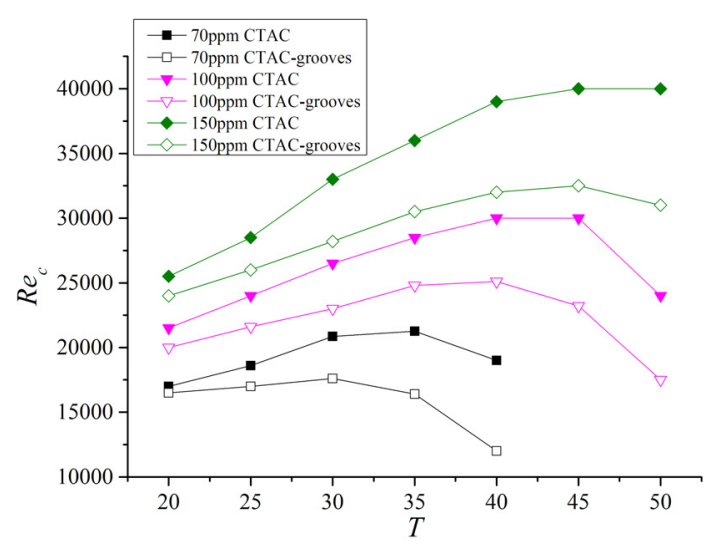

Figure 4: Critical Reynolds number versus temperature at different CTAC concentrations.

Furthermore, the critical Reynolds numbers and critical temperatures also increase with the rise of surfactant concentration whether the channel is smooth or grooved. The critical temperatures at different concentrations are listed in Table 1. It is speculated that the SIS are easier to form and harder to be destroyed with increasing concentration, resulting in the rise of $\operatorname{Re}_{c}$ and $T_{c}$. This speculation is further verified by the experimental results obtained by Hadri et al. (2011) and Wei et al. (2012). This feature of lower critical temperature in the grooved channel is beneficial for the application in the field of heat transfer. The drag-reducing effect of surfactant could decrease and the heat transfer performance could rise if temperature slightly exceeds $T_{c}$. Moreover, lower $T_{c}$ temperature ensures its feasibility in practice and a high potential value in the field of heat transfer.
Table 1: The critical temperatures at different CTAC concentrations.

\begin{tabular}{|c|c|c|c|}
\hline C/ppm & $\mathbf{7 0}$ & $\mathbf{1 0 0}$ & $\mathbf{1 5 0}$ \\
\hline Smooth channel & $35^{\circ} \mathrm{C}$ & $45^{\circ} \mathrm{C}$ & $\geq 50^{\circ} \mathrm{C}$ \\
Grooved channel & $30^{\circ} \mathrm{C}$ & $40^{\circ} \mathrm{C}$ & $45^{\circ} \mathrm{C}$ \\
\hline
\end{tabular}

Figure 5 shows the drag-reducing performance of $0-150$ ppm CTAC solutions in the smooth and grooved channels at $20{ }^{\circ} \mathrm{C}$. It can be seen that there is no drag-reducing effect in the entire range of experimental Reynolds number for the grooves without surfactant. However, Bechert et al. (1997) obtained the drag-reduction effect when $s^{+}$was smaller than 27 with almost the same shape and dimensionless size of grooves (riblet) by using a precise measurement. There is a discrepancy between his results and ours. Detailed analysis shows that the discrepancy may mainly be caused by different smoothness of the grooved surfaces. In their study, the riblet surfaces were relatively smooth by using the plastic riblet film (Bechert et al., 1997). However, the grooved surfaces used in the present study are manufactured directly by a milling machine. The small size of the grooves makes it impossible to completely remove some small burr structures and some accidental machining errors may occur during the machining process, resulting in a relatively poor smoothness of the grooved surface (seen from Figure 2). So the friction factor is larger than that obtained by Bechert et al. (1997) under the same conditions, resulting in the disappearance of the drag-reducing phenomenon at low Reynolds numbers in the present study. Nevertheless, in practical industrial pipeline transportation, the pipeline system constructed mainly from steel pipes will undergo corrosion as time goes by and be subject to deposition of fouling on its surface, and finally leading to development of roughness. Thus the less-smooth grooved surfaces used in this study are more approximate to the conditions of industrial grooved pipes after long-term use, and therefore the present results can be a guidance for the practical application of grooved pipe.

According to the studies on the flow in rough pipes by Virk (1971), Bewersdorff et al. (1993), Yang \& Dou (2010) and Różański (2011), the dragreducing phenomenon could not be found in rough pipes for water and similar drag-increasing results also occured. So the present grooves seem to be merely regarded as a "rough" wall. Moreover, their studies (Virk, 1971; Bewersdorff et al., 1993; Yang and Dou, 2010; Różański, 2011) on the drag reduction of polymer or surfactant solutions in rough pipes 
showed that the drag reduction effects were lower in rough pipes than in smooth pipes for both polymer and surfactant solutions. However, the phenomenon that the longitudinal grooves can enhance drag reduction is found in the present experiment based on drag-reducing surfactant solution. This phenomenon will be discussed later. Moreover, Koury and Virk (1995) carried out an investigation on the drag reduction of polymer in a pipe lined with longitudinal riblets and found that longitudinal riblets could enhance the drag reduction of polymer, the conclusion of which is similar to ours. It becomes evident that the longitudinal grooved surface used in this experiment should not be merely regarded as a "rough" wall, because it still remains the character of the restricted turbulent vortex, although it cannot reduce drag for water. It shows a character of "rough" wall due to a larger size of groove and relatively poor smoothness of the grooved surface. This result verifies the conclusion that grooved surfaces could be considered as transitionally rough under certain conditions (Tani, 1988; Saravi and Cheng, 2013).

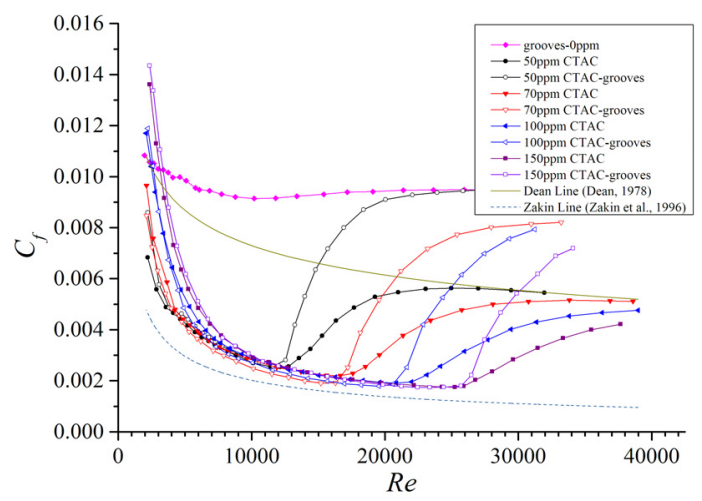

Figure 5: Drag reduction performance of surfactant in the smooth and grooved channels at $20{ }^{\circ} \mathrm{C}$. The Dean line and Zakin line are from Dean (1978) and Zakin et al. (1996), respectively.

Grooves decrease the drag by restricting the spanwise motions of turbulent vortices near the wall. In order to achieve drag reduction, the scale of the turbulent vortex should correspond to the dragreducing scale range of grooves (Bechert et al., 1986). For water, the turbulence is a complicated system with a large number of multi-scale turbulent eddies, the sizes of which still remain undetermined. It can be seen from other literature that the scale of a turbulent vortex near the wall is approximately 20-40 wall units while vortices of 3-4 wall units also exist (Adrian et al., 2000). However, for the grooves used in this experiment, its scale $s^{+}$extends with increas- ing Reynolds number. The range of the grooved scale in this study is larger than 12. Because the grooved scale is similar to the scale of turbulent vortex near the wall, the grooves cannot restrict effectively the motions of turbulent vortices, and thus the smaller turbulent vortices could intrude into the valley of grooves and scour the sidewall, representing an increase in frictional area and drag. By comparing the grooved scale with the turbulent vortex scale, it is speculated that the former is smaller than the latter when the drag reduction can be achieved, but the accurate drag-reducing scale range of grooves still needs to be determined by further experiments. Lee et al. (2001) conducted a study on the turbulent structures over the longitudinal semi-circular riblet surface by the particle image velocity (PIV) technique. They found that the longitudinal riblets could restrict the spanwise motions of streamwise turbulent vortices near the wall when riblets reduced drag at a lower Reynolds number, and discovered the phenomenon that turbulent vortices intruded into the valley of grooves and scour the sidewall when the drag-increasing case occurred at a higher Reynolds number. To some extent, their results could verify the reliability of the experimental results and analysis in the present study.

Comparing the curves for CTAC solutions in the smooth channel with that in the grooved channel, we find that the drag reduction effectiveness of surfactant solutions in the smooth channel is better than that in the grooved one for $50 \mathrm{ppm}$; however, it is just the opposite for 70-150 ppm CTAC solutions. This is an exciting discovery. In order to explain the above phenomenon easily, four effects of grooves should be defined in the present study as follows:

(1) The "positive restriction effect"- grooves can decrease the drag by restricting the spanwise motions of turbulent vortices near the wall, when the turbulent vortex scale matches up with the scale of grooves at a lower Reynolds number.

(2) The "negative restriction effect"- grooves cannot restrict the spanwise motions of turbulent vortices near the wall and the smaller turbulent vortices could intrude into the valley of grooves and scour the sidewall at a higher Reynolds number, resulting in an increase in drag.

The existence of the above two effects have been verified by Lee et al. (2001) as mentioned above.

(3) The "positive peak effect"- the grooved tip could promote the formation of SIS by providing a larger shear stress at a lower Reynolds number.

(4) The "negative peak effect"- the grooved tip destroys the formation of SIS by a larger shear stress, especially at a higher Reynolds number. 
The influence of shear stress on the SIS in a surfactant solution was reported in the literature by Zhou et al. (2011) and Qi et al. (2011), and the characteristic of a larger shear stress at grooved tips was reported in the literature by Goldstein et al. (1995) and Zhang et al. (2011). To some extent, these previous investigations could provide the basis for the possibility of these two "peak effects" mentioned above. However, it is noted that these two "peak effects" are speculative in the present study, and need further investigation to verify them. These four effects above will be summarized to explain the results obtained in the present experiment and the interaction between grooves and surfactant solutions in a simpler way.

For $50 \mathrm{ppm}$ surfactant solution, the number of surfactant molecule is too few due to the lower surfactant concentration, resulting in a weaker SIS and "positive peak effect". Moreover, the weaker SIS cannot inhibit the formation of small turbulent vortices effectively. Therefore, the turbulent vortex scale is smaller than the grooved scale, leading to the "negative restriction effect" of grooves. Furthermore, grooves exhibit a larger "negative peak effect" at a higher Reynolds number. As a result, the overall drag-reducing effectiveness is weakened.

In contrast, for $70 \mathrm{ppm}$ or higher concentrations, the "positive peak effect" is intensified with increasing numbers of surfactant molecule at a lower Reynolds number. Moreover, the SIS is more effective to inhibit the formation of small turbulent vortex, resulting in an increasing scale of turbulent vortex, so the "positive restriction effect" is obtained. As a result, drag reduction effectiveness of surfactant solution in the grooved channel is enhanced by the interaction of the two effects before the critical Reynolds number $R_{c}$.

Figure 5 also shows that the drag-reducing enhancement effect is the best for $70 \mathrm{ppm}$, and then decreases in the order $100 \mathrm{ppm}, 150 \mathrm{ppm}$ and $50 \mathrm{ppm}$. For the $50 \mathrm{ppm}$ case, there is no enhancement effect. This indicates that the drag-reducing enhancement effect becomes weaker with increasing surfactant concentration. In summary, the "restriction effect" and the "peak effect" of grooves change with Reynolds number and concentration of surfactant solution, and the drag reduction effectiveness of surfactant solution in a grooved channel depends on the interaction of the two effects. This implies that surfactant and grooves reduce drag by separate mechanisms, and they can enhance drag reduction by mutual promotion, that is to say, there is a synergistic effect, which expands the range of applications and their potential applications. The possible synergistic mechanism is that, in the surfactant solution, the scale of turbulent vortex becomes larger due to the inhibition of SIS compared with that in water, and grooves can restrict the penetration of the larger vortices into the grooved valley more effectively at the appropriate surfactant concentration and Reynolds number, and thus fewer turbulent vortices scour the grooved surface and the momentum exchange decreases compared with the smooth surface. Moreover, the larger shear stress at the grooved tips can make the formation of the nearwall SIS easier before the $\mathrm{Re}_{c}$. As a result, friction drag can be reduced again by grooves on the basis of the drag-reducing surfactant solution. For the grooves used in this study, the optimal concentration of surfactant is $70 \mathrm{ppm}$, the drag-reducing effect of which is enhanced by grooves by $5 \%$ on the basis of drag-reducing surfactant solution (the maximum drag reduction rate is about $66 \%$ for $70 \mathrm{ppm}$ solution without grooves, and it is about $71 \%$ for the combination of surfactant and grooves at $20{ }^{\circ} \mathrm{C}$ ). In order to verify that the enhancement effect of $5 \%$ is not caused by the uncertainty of measurements, an uncertainty analysis is used as an explanation. For example, the maximum enhancement effect is obtained at $\operatorname{Re} \approx 15200$ for $70 \mathrm{ppm}$, and the measurement uncertainty of DR can be calculated by using Eq. (6), Eq. (7) and the relevant data (such as the measurement uncertainty of flux and pressure drop, the pressure drop, flux, etc. at this time). They are about $1.1 \%$ and $1 \%$ for smooth and grooved channel respectively, thus the maximum measurement uncertainty of DR between smooth and grooved channel is about $2.1 \%$, indicating that the enhancement effect is valid.

It can also be found from Figure 5 that, at the same concentration of surfactant solution, the friction factor rises much more rapidly in the grooved channel than in the smooth channel when Re is larger than $R_{\mathrm{c}}$. It is understandable that the "negative peak effect" of grooves is too strong to destroy the SIS more quickly, especially at a higher Reynolds number, and thus the friction factor increases faster in the grooved channel. The mutual inhibition between drag-reducing effect and heat transfer effect of surfactant may benefit its application in the field of heat transfer. The surfactant solution can enhance heat transfer performance and reduce drag reduction performance quickly in a groove channel by increasing the Reynolds number slightly over $\mathrm{Re}_{\mathrm{c}}$ in the heat transfer system. Furthermore, the drag reduction enhancement performance of grooves in surfactant solution can be used in pipeline transportation. Though 
the four effects of grooves mentioned above cannot be observed directly in this experiment, they can be verified indirectly by other researchers' discoveries (Zhou et al., 2011; Qi et al., 2011; Goldstein et al., 1995; Zhang et al., 2011). It is understandable that these four effects of grooves are used to explain our results. Unfortunately, in the present experiment, because of the rough and poor light transmittance of the grooved surface manufactured directly by a milling machine, it is not appropriate to measure by the particle image velocimetry (PIV) technique and it is difficult to accurately measure the turbulence structures of the cross section near the grooves due to the small size of the groove. Thus, our speculations cannot be verified directly by the present experimental results due to the absence of corresponding visualization results. In order to verify directly the validity of the conjecture, additional studies such as a direct numerical simulation (DNS) method or other effective measurement techniques are necessary to visualize the turbulent structures of drag-reducing surfactant solution over the longitudinal grooved surface. The next step of this study is to make a qualitative research and try to verify the conjecture of this paper by the DNS method in the future.

\section{Drag Reduction Rate (DR)}

Drag reduction versus Reynolds numbers for surfactant solutions in the smooth channel or grooved channel at different temperatures and concentrations are respectively presented in Figures 6-8. Drag reduction rates (DR) at different conditions are calculated by Eqs. (2)-(4), separately. It can be seen from Figures 6(a), 7(a) and 8(a) that, for a certain surfactant concentration in the smooth channel, the maximum drag reduction rate $\left(\mathrm{DR}_{\max }\right)$ grows with increasing temperature until the temperature reaches the point of $T_{c}$, and the comparison of the three concentrations proves that the range of maximum drag reduction rate increases with surfactant concentration. For $70 \mathrm{ppm}, \mathrm{DR}_{\max }=66-69 \%$; for $100 \mathrm{ppm}$, $\mathrm{DR}_{\max }=68-71 \%$; and for $150 \mathrm{ppm}, \mathrm{DR}_{\max }=70-74 \%$. Before the temperature rises to $T_{c}$, the thermal Brownian motions of molecules intensify, and the number of surfactant molecules rises with increasing surfactant concentration. Both of them can make the formation of SIS easier and stronger, and thus the inhibition effect on turbulence is enhanced, resulting in the slight increase in DR. This finding corresponds to the result obtained by Wei et al. (2012).

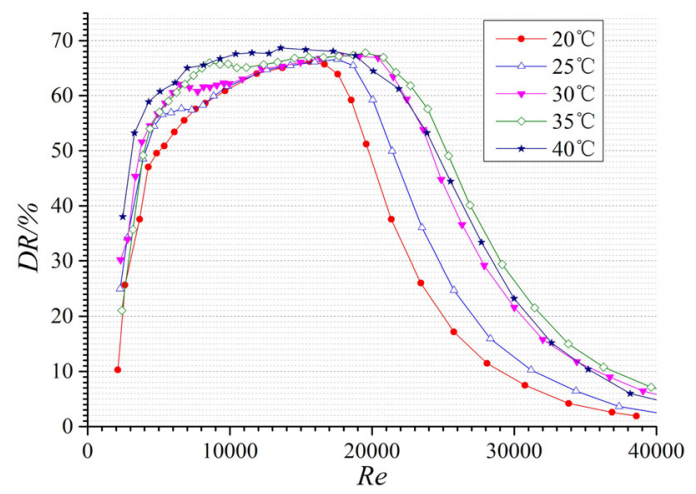

(a)

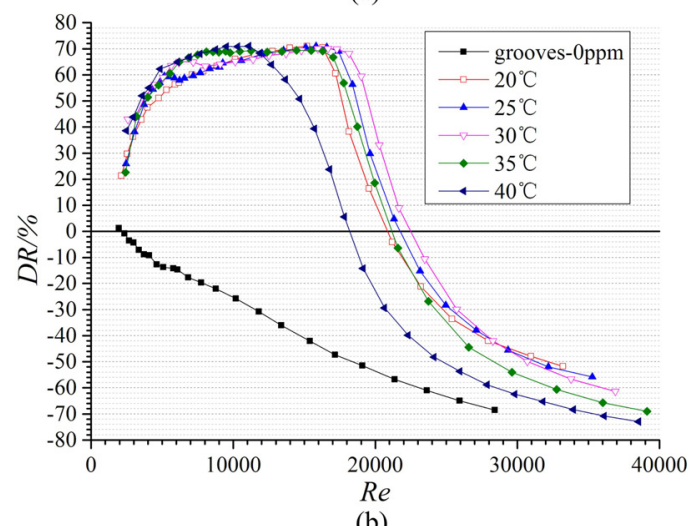

(b)

Figure 6: Drag reduction versus Reynolds numbers for 70 ppm CTAC. (a) Smooth channel. (b) Grooved channel. 


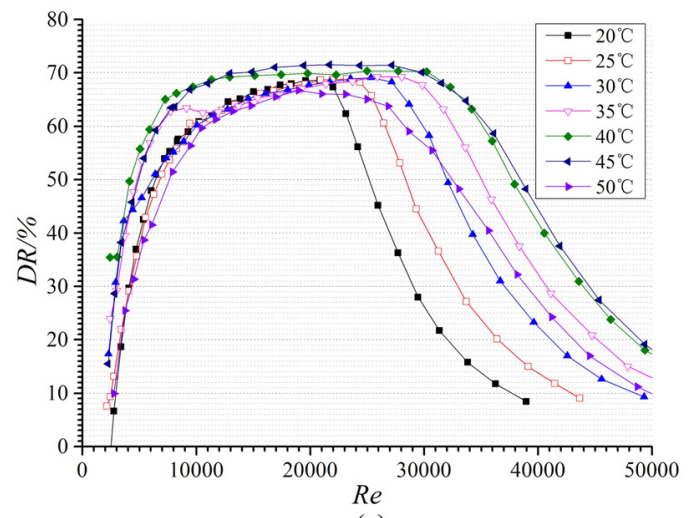

(a)

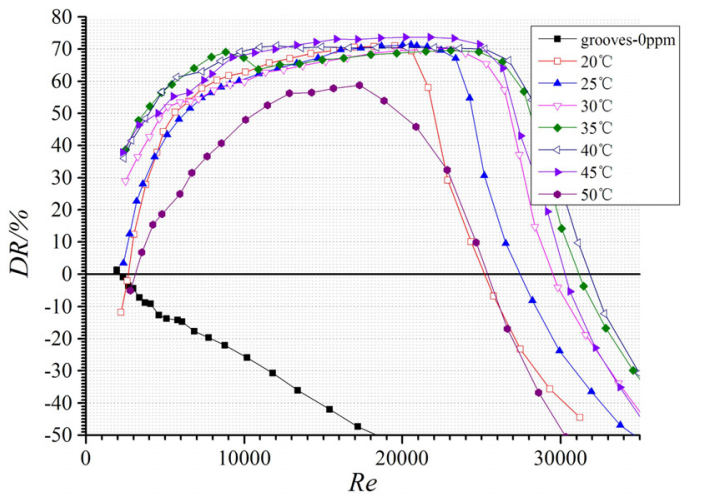

(b)

Figure 7: Drag reduction versus Reynolds numbers for 100 ppm CTAC. (a) Smooth channel. (b) Grooved channel.

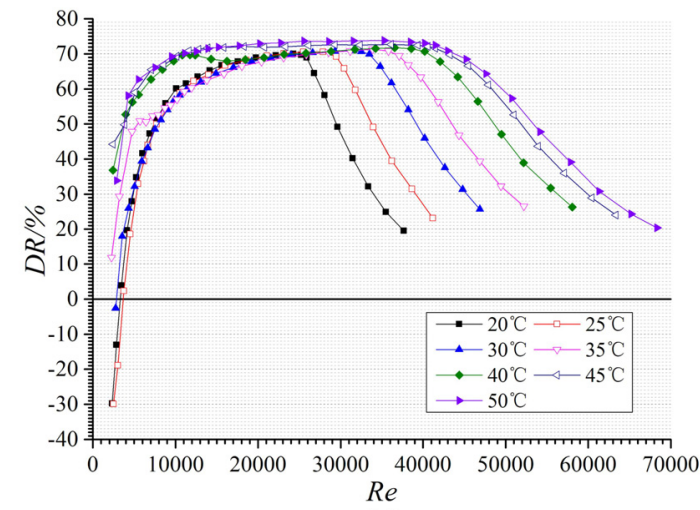

(a)

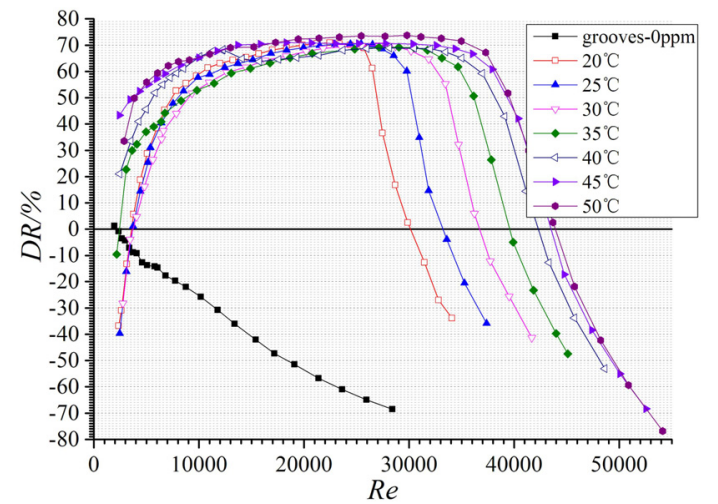

(b)

Figure 8: Drag reduction versus Reynolds numbers for 150 ppm CTAC. (a) Smooth channel. (b) Grooved channel.

However, there exists an interesting phenomenon. It can be found from Figures 6(b), 7(b) and 8(b) that the temperature and surfactant concentration have little influence on the maximum drag reduction rate of surfactant solution in the grooved channel before $\mathrm{T}_{\mathrm{c}}$, and the $\mathrm{DR}_{\max }$ is about $70 \%$ (it can reach about $73 \%$ only occasionally at a temperature higher than $\mathrm{T}_{\mathrm{c}}$ ). This can be explained by the fact that, when the temperature and concentration are lower, the surfactant solution itself has a lower drag reduction rate, the "positive peak effect" and the "positive restriction effect" of grooves are thus more effective (refer to the analysis on the four effects of grooves), resulting in a higher drag reduction rate $\left(\mathrm{DR}_{\max } \approx 70 \%\right)$. However, with the increase of temperature and concentration, the surfactant solution itself has a higher drag reduction rate because the SIS is easier to form. Therefore, the "positive peak effect" and the "positive restriction effect" of grooves are inhibited, also resulting in the total drag reduction rate of about $70 \%$. With further increase of temperature and concentration, these two effects disappear. As a result, the maximum drag reduction rate might only exhibit the maximum drag reduction rate of the surfactant solution itself or even be weakened slightly.

\section{CONCLUSIONS}

In the present study, the complementarity of drag reducing mechanisms between surfactant and grooves was confirmed by experimentally investigating the drag-reducing performance of surfactant solutions in smooth and grooved channels, respectively. The conclusions can be summarized as follows:

1. The surfactant solution has an excellent drag reduction in both smooth and grooved channels. Except for $50 \mathrm{ppm}$ solution, the drag-reducing effect of surfactant solution is enhanced by grooves, but the enhancement effect decreases with increasing surfactant concentration, and furthermore, at a higher concentration, even the total drag reduction effect is 
weakened. This comes from the interaction between the "restriction effect" and the "peak effect" of grooves. It is noted that the "restriction effect" and the "peak effect" may be the positive or negative effect at different Reynolds numbers or surfactant concentrations, and the "peak effect" of grooves has no drag reduction enhancement effect in the Newtonian fluids compared with surfactant solution. For the grooves used in this study, the optimal concentration of surfactant is $70 \mathrm{ppm}$, the drag-reducing effect of which is enhanced by grooves by $5 \%$ on the basis of drag-reducing surfactant solution at $20^{\circ} \mathrm{C}$.

2. The critical temperature and critical Reynolds number of surfactant solution are smaller in the grooved channel than in the smooth channel. Moreover, the friction factor in grooved channel rises much more rapidly than that in the smooth channel when $\mathrm{Re}$ is larger than $\mathrm{Re}_{c}$. These two features may contribute to the application in the field of heat transfer, especially in the pipeline system with fluid transportation and heat transfer. The combination of surfactants and grooves can either enhance the dragreducing effect in fluid transportation or reinforce the heat transfer effect in the heat exchanger, which expands the range of their potential applications.

3. The temperature and surfactant concentration have little influence on the maximum drag reduction rate of surfactant solution in the grooved channel before $T_{c}$, which differs from the surfactant solution in the smooth channel.

\section{ACKNOWLEDGEMENT}

The present work is supported by National Natural Science Foundation of China (No. 51225601).

\section{NOMENCLATURE}

$\mathrm{C}_{\mathrm{f}} \quad$ Fanning friction factor of water in a smooth channel, (-)

D Equivalent diameter, (m)

DR Drag reduction rate, (-)

$\mathrm{DR}_{\max }$ Maximum drag reduction rate, (-)

$\mathrm{h} \quad$ Depth of grooves, $(\mathrm{m})$

$\mathrm{H} \quad$ Channel height, (m)

L Distance of two pressure taps, (m)

Re Reynolds number, (-)

$\mathrm{Re}_{\mathrm{c}} \quad$ Critical Reynolds number, (-)

$\mathrm{s} \quad$ Spacing of grooves, $(\mathrm{m})$

$\mathrm{s}^{+} \quad\left(u_{\tau} s\right) / v,(-)$

$\mathrm{T} \quad$ Temperature, $\left({ }^{\circ} \mathrm{C}\right)$

$$
\begin{array}{ll}
\mathrm{T}_{\mathrm{c}} & \text { Critical temperature, }\left({ }^{\circ} \mathrm{C}\right) \\
u_{\tau} & \text { Frictional velocity, }\left(\mathrm{m} \cdot \mathrm{s}^{-1}\right) \\
\mathrm{U}_{\mathrm{b}} & \text { Bulk mean velocity, }\left(\mathrm{m} \cdot \mathrm{s}^{-1}\right) \\
\mathrm{W} & \text { Channel width, }(\mathrm{m})
\end{array}
$$

\section{Greek Letters}

$\begin{array}{ll}\alpha & \text { Angle of grooved tip, }(\mathrm{rad}) \\ v & \text { Kinetic viscosity of solvent, }\left(\mathrm{m}^{2} \cdot \mathrm{s}^{-1}\right) \\ \rho & \text { Density of solvent, }\left(\mathrm{kg} \cdot \mathrm{m}^{-3}\right) \\ \tau_{\mathrm{w}} & \text { Wall shear stress, }\left(\mathrm{N} \cdot \mathrm{m}^{-2}\right) \\ \Delta P & \text { Pressure drop, }(\mathrm{Pa})\end{array}$

\section{Subscripts}

$\begin{array}{ll}\text { g } & \text { Groove } \\ \text { gs } & \text { Groove and surfactant } \\ \text { s } & \text { Surfactant }\end{array}$

\section{REFERENCES}

Adrian, R. J., Christensen, K. T. and Liu, Z. C., Analysis and interpretation of instantaneous turbulent velocity fields. Experiments in Fluids, 29(3), 275 (2000).

Al-Wahaibi, T., Al-Wahaibi, Y., Al-Ajmi, A., Yusufa, N., Al-Hashmia, A. R., Olawaleb, A. S. and Mohammedb, I. A., Experimental investigation on the performance of drag reducing polymers through two pipe diameters in horizontal oilwater flows. Experimental Thermal and Fluid Science, 50, 139 (2013).

Bechert, D. W., Bartenwerfer, M., Hoppe, G. and Reif, W. E., Drag reduction mechanisms derived from shark skin. American Institute of Aeronautics and Astronautics, New York, 2, 1044 (1986).

Bechert, D. W., Bruse, M., Hage, W., Van der Hoeven, J. G. T. and Hoppe, G., Experiments on dragreducing surfaces and their optimization with an adjustable geometry. Journal of Fluid Mechanics, 338, 59 (1997).

Bewersdorff, H. W. and Ohlendorf, D., The behaviour of drag-reducing cationic surfactant solutions. Colloid and Polymer Science, 266(10), 941 (1988).

Bewersdorff, H. W. and Thiel, H., Turbulence structure of dilute polymer and surfactant solutions in artificially roughened pipes. Applied Scientific Research, 50(3-4), 347 (1993).

Burger, E. D., Munk, W. R. and Wahl, H. A., Flow increase in the Trans Alaska pipeline through use of a polymeric drag-reducing additive. Journal of Petroleum Technology, 34(2), 377 (1982). 
Chamorro, L. P., Arndt, R. E. A. and Sotiropoulos F., Drag reduction of large wind turbine blades through riblets: Evaluation of riblet geometry and application strategies. Renewable Energy, 50, 1095 (2013).

Choi, K. S., Drag Reduction Mechanisms and NearWall Turbulence Structure with Riblets. Structure of Turbulence and Drag Reduction, Springer Berlin Heidelberg, p. 553 (1990).

Dean, R. B., Reynolds number dependence of skin friction and other bulk flow variables in twodimensional rectangular duct flow. Journal of Fluids Engineering, 100(2), 215 (1978).

Debye, P. and Anacker, E. W., Micelle shape from dissymmetry measurements. The Journal of Physical Chemistry, 55(5), 644 (1951).

Goldstein, D., Handler, R. and Sirovich, L., Direct numerical simulation of turbulent flow over a modeled riblet covered surface. Journal of Fluid Mechanics, 302, 333 (1995).

Hadri, F., Besq, A., Guillou, S. and Makhloufi, R., Temperature and concentration influence on drag reduction of very low concentrated CTAC/NaSal aqueous solution in turbulent pipe flow. Journal of Non-Newtonian Fluid Mechanics, 166(5), 326 (2011).

Kawaguchi, Y., Segawa, T., Feng, Z. and Li, P., Experimental study on drag-reducing channel flow with surfactant additives-spatial structure of turbulence investigated by PIV system. International Journal of Heat and Fluid Flow, 23(5), 700 (2002).

Koury, E. and Virk, P. S., Drag reduction by polymer solutions in a riblet-lined pipe. Applied Scientific Research, 54(4), 323 (1995).

Lee, S. J. and Lee, S. H., Flow field analysis of a turbulent boundary layer over a riblet surface. Experiments in Fluids, 30(2), 153 (2001).

Li, F. C., Kawaguchi, Y., Hishida, K. and Oshima, M., Investigation of turbulence structures in a drag-reduced turbulent channel flow with surfactant additive by stereoscopic particle image velocimetry. Experiments in Fluids, 40(2), 218 (2006).

Moaven, K., Rad, M. and Taeibi-Rahni, M., Experimental investigation of viscous drag reduction of superhydrophobic nano-coating in laminar and turbulent flows. Experimental Thermal and Fluid Science, 51, 239 (2013).

Mysels, K. J., Flow of thickened fluids. U.S. Patent 2,492,173 (1949).

Nikuradse, J., Laws of flow in rough pipes. Washington: National Advisory Committee for Aeronautics (1950).
Ohlendorf, D., Interthal, W. and Hoffmann, H., Surfactant systems for drag reduction: Physicochemical properties and rheological behavior. Rheologica Acta, 25(5), 468 (1986).

Pang, M., Wei, J. and Yu, B., Effect of particle clusters on turbulence modulations in liquid flow laden with fine solid particles. Brazilian Journal of Chemical Engineering, 28(3), 433 (2011).

Qi, Y., Kesselman, E., Hart, D. J., Talmon, Y., Mateo, A. and Zakin, J. L., Comparison of oleyl and elaidyl isomer surfactant-counterion systems in drag reduction, rheological properties and nanostructure. Journal of Colloid and Interface Science, 354(2), 691 (2011).

Quintavalla, S. J., Angilella, A. J. and Smits, A. J., Drag reduction on grooved cylinders in the critical Reynolds number regime. Experimental Thermal and Fluid Science, 48, 15 (2013).

Rehage, H., Wunderlich, I. and Hoffmann, H., Shear induced phase transitions in dilute aqueous surfactant solutions. Polymers as Colloid Systems, Steinkopff, p. 51-59 (1985).

Różański, J., Flow of drag-reducing surfactant solutions in rough pipes. Journal of Non-Newtonian Fluid Mechanics, 166(5), 279 (2011).

Saravi, S. S. and Cheng, K., A review of drag reduction by riblets and micro-textures in the turbulent boundary layers. European Scientific Journal, 9(33) (2013).

Tani, I., Drag reduction by riblet viewed as roughness problem. Proceedings of the Japan Academy, Ser. B, Physical and Biological Sciences, 64(2), 21 (1988).

Toms, B. A., Some observations on the flow of linear polymer solutions through straight tubes at large Reynolds numbers. Proceedings of the 1st International Congress on Rheology, 2, 135 (1948).

Virk, P. S., Drag reduction in rough pipes. Journal of Fluid Mechanics, 45(2), 225 (1971).

Walsh, M. J., Drag Characteristics of V-Groove and Transverse Curvature Riblits. AIAA Prog. Astronaut. Aeronaut., 72, 168 (1980).

Walsh, M. J. and Lindemann, A. M., Optimization and application of riblets for turbulent drag reduction. American Institute of Aeronautics and Astronautics (1984).

Walsh, M. J., Turbulent boundary layer drag reduction using riblets. AIAA, Aerospace Sciences Meeting, 1 (1982).

Wei, J., Wang, J., Zhang, C. and Kawaguchi, Y., Combined effects of temperature and Reynolds number on drag-reducing characteristics of a cationic surfactant solution. The Canadian Journal of Chemical Engineering, 90(5), 1304 (2012). 
Yang, S. Q. and Dou, G., Turbulent drag reduction with polymer additive in rough pipes. Journal of Fluid Mechanics, 642, 279 (2010).

Zakin, J. L., Myska, J. and Chara, Z., New limiting drag reduction and velocity profile asymptotes for nonpolymeric additives systems. AIChE Journal, 42(12), 3544 (1996).

Zhang, D. Y., Luo, Y. H., Xiang, L. I. and Chen, H. W., Numerical simulation and experimental study of drag-reducing surface of a real shark skin. Journal of Hydrodynamics, Ser. B, 23(2), 204 (2011).

Zhou, Y. B., Xu, N., Ma, N., Li, F. C., Wei, J. J. and Yu, B., On Relationships among the aggregation number, rheological property, and turbulent dragreducing effect of surfactant solutions. Advances in Mechanical Engineering, 3, 345328 (2011). 